PROCEEDINGS OF THE

AMERICAN MATHEMATICAL SOCIETY

Volume 129, Number 8, Pages 2239-2243

S 0002-9939(01)05500-9

Article electronically published on March 15, 2001

\title{
INEQUALITIES FOR PRODUCTS OF SPECTRAL RADII
}

\author{
M. OMLADIČ, H. RADJAVI, P. ROSENTHAL, AND A. SOUROUR
}

(Communicated by David R. Larson)

\begin{abstract}
It is shown that submultiplicative inequalities for spectral radii often imply supermultiplicative inequalities, and vice versa.
\end{abstract}

\section{INTRODUCTION}

The spectral radius of a linear transformation on a finite-dimensional complex space, or of a bounded linear operator on a complex Banach space, or, more generally, of an element of a complex Banach algebra, is the supremum of the moduli of numbers in the spectrum of the element. The famous spectral radius formula of Gelfand states that the spectral radius, $r(A)$, is given by

$$
r(A)=\lim _{n \rightarrow \infty}\left\|A^{n}\right\|^{\frac{1}{n}} .
$$

In this note we consider semigroups (i.e., subsets closed under multiplication) of operators or elements of a Banach algebra on which the spectral radius is $k$ submultiplicative, in the sense that there is a positive real number $k$ such that $r(A B) \leq k r(A) r(B)$ for all $A$ and $B$ in the semigroup. Our main results give sufficient conditions under which $k$-submultiplicativity implies that

$$
r(A B) \geq \frac{1}{k} r(A) r(B) \quad \text { for all } A \text { and } B .
$$

\section{A general Result in Banach algebras}

Recall that $r(A B)=r(B A)$ for any elements of a Banach algebra (in fact, the spectra of $A B$ and $B A$ differ at most by $\{0\}$ ). Our basic lemmas hold for any non-negative functions agreeing on $A B$ and $B A$.

Lemma 1. If $k>0$ and $f$ is a non-negative function on a semigroup satisfying $f(A B)=f(B A)$ and $f(A B) \leq k f(A) f(B)$ for all $A, B$, then $f\left(A^{n} B^{n}\right) \leq$ $k^{n-1}(f(A B))^{n}$ for every positive integer $n$.

Received by the editors August 12, 1998 and, in revised form, February 26, 1999.

2000 Mathematics Subject Classification. Primary 47A10, 46H05.

(C)2001 American Mathematical Society 
Proof. The proof is by induction on $n$. The case $n=1$ is trivial, so assume the inequality for $n-1$. Then

$$
\begin{aligned}
f\left(A^{n} B^{n}\right) & =f\left(A A^{n-1} B^{n-1} B\right) \\
& =f\left(A^{n-1} B^{n-1} B A\right) \\
& \leq k f\left(A^{n-1} B^{n-1}\right) f(B A) \\
& \leq k k^{n-2}(f(A B))^{n-1} f(A B) \\
& =k^{n-1}(f(A B))^{n} .
\end{aligned}
$$

Lemma 2. If $\varepsilon>0$ and $f$ is a non-negative function on a semigroup satisfying $f(A B)=f(B A)$ and $f(A B) \geq \varepsilon f(A) f(B)$ for all $A, B$, then $f\left(A^{n} B^{n}\right) \geq$ $\varepsilon^{n-1}(f(A B))^{n}$ for every positive integer $n$.

Proof. The proof of this lemma is exactly the same as the preceding one except that the inequalities are reversed.

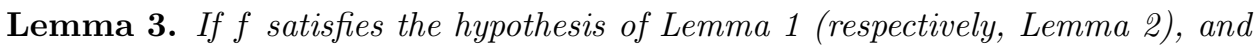
if $A$ and $B$ are elements of the semigroup such that $\left\{f\left(A^{n} B^{n}\right)\right\}$ is bounded and bounded away from 0 , then $f(A B) \geq \frac{1}{k}$ (respectively, $f(A B) \leq \frac{1}{\varepsilon}$ ).

Proof. Suppose $f$ satisfies the hypothesis of Lemma 1 . Choose a subsequence so that $f\left(A^{n_{i}} B^{n_{i}}\right)$ converges to some $t$; then $t>0$. Since

$$
f\left(A^{n_{i}} B^{n_{i}}\right) \leq k^{n_{i}-1}(f(A B))^{n_{i}},
$$

taking $n_{i}$ th roots gives

$$
\left(f\left(A^{n_{i}} B^{n_{i}}\right)\right)^{\frac{1}{n_{i}}} \leq k^{1-\frac{1}{n_{i}}} f(A B) .
$$

Taking the limit as $\left\{n_{i}\right\} \rightarrow \infty$ yields

$$
1 \leq k f(A B) .
$$

The proof of the consequence of Lemma 2 is exactly the same except that the inequalities are reversed.

Theorem 1. If $\mathcal{S}$ is a semigroup contained in a Banach algebra and there exist $\varepsilon>0$ and $k>0$ such that $\operatorname{cr}(A) r(B) \leq r(A B) \leq k r(A) r(B)$ for all $\{A, B\} \subset \mathcal{S}$, then

for all $\{A, B\} \subset \mathcal{S}$.

$$
\frac{1}{k} r(A) r(B) \leq r(A B) \leq \frac{1}{\varepsilon} r(A) r(B)
$$

Proof. The inequalities of the hypothesis clearly extend to the semigroup $\mathbb{R}^{+} \mathcal{S}=$ $\{t S: t \geq 0, S \in \mathcal{S}\}$.

Fix $A$ and $B$ in $\mathbb{R}^{+} \mathcal{S}$; since multiplying each of $A$ and $B$ by positive numbers does not change any of the inequalities, we can assume that $r(A)=r(B)=1$. We must then show that $\frac{1}{k} \leq r(A B) \leq \frac{1}{\varepsilon}$.

By Lemma 3, it suffices to show that $r\left(A^{n} B^{n}\right)$ is bounded and is bounded away from 0 .

By hypothesis,

$$
\varepsilon r\left(A^{n}\right) r\left(B^{n}\right) \leq r\left(A^{n} B^{n}\right) \leq k r\left(A^{n}\right) r\left(B^{n}\right)
$$


for all $n$. Since $r(A)=r(B)=1, r\left(A^{n}\right)=r\left(B^{n}\right)=1$, and

$$
\varepsilon \leq r\left(A^{n} B^{n}\right) \leq k
$$

for all $n$.

Corollary 1. Let $\mathcal{S}$ be a semigroup contained in a Banach algebra. If $r$ is submultiplicative on $\mathcal{S}$ and if there is an $\varepsilon>0$ such that $r(A B) \geq \operatorname{\varepsilon r}(A) r(B)$ for all $A$ and $B$ in $\mathcal{S}$, then $r$ is multiplicative on $\mathcal{S}$.

Proof. Submultiplicativity means that $k=1$ in the hypothesis of Theorem 1 and $\frac{1}{1}=1$.

\section{LiNEAR TRANSFORMATIONS ON FINITE-DIMENSIONAL SPACES}

In the finite-dimensional case, the existence of an $\varepsilon$ such that $r(A B) \geq \varepsilon r(A) r(B)$ can be inferred under various hypotheses.

Theorem 2. Let $\mathcal{S}$ be a semigroup of linear transformations on a finite-dimensional space which is closed under multiplication by positive real numbers and is also closed in the topological sense. If $\mathcal{S}$ has no non-zero divisors and $r(A B) \leq k r(A) r(B)$ for all $A$ and $B$ in $\mathcal{S}$, then $r(A B) \geq \frac{1}{k} r(A) r(B)$ for all $A$ and $B$ in $\mathcal{S}$.

Proof. This will follow from Theorem 1 if we show the existence of an $\varepsilon>0$ such that $r(A B) \geq \varepsilon r(A) r(B)$, for every $A$ and $B$ in $S$. If there were no such $\varepsilon$, then for every positive integer $n$ there would be $A_{n}$ and $B_{n}$ in $\mathcal{S}$ satisfying

$$
r\left(A_{n} B_{n}\right)<\frac{1}{n} r\left(A_{n}\right) r\left(B_{n}\right) .
$$

Then

$$
r\left(\frac{A_{n}}{\left\|A_{n}\right\|} \frac{B_{n}}{\left\|B_{n}\right\|}\right)<\frac{1}{n} r\left(\frac{A_{n}}{\left\|A_{n}\right\|}\right) r\left(\frac{B_{n}}{\left\|B_{n}\right\|}\right) .
$$

Since the unit sphere of the space of linear transformations is compact, there are transformations $E$ and $F$ and an increasing sequence of positive integers $\left\{n_{i}\right\}$ such that $\left\{\frac{A_{n_{i}}}{\left\|A_{n_{i}}\right\|}\right\} \rightarrow E$ and $\left\{\frac{B_{n_{i}}}{\left\|B_{n_{i}}\right\|}\right\} \rightarrow F$. It follows that $r(E F)=0$ (spectral radius is continuous in finite-dimensions). Since $E F$ is nilpotent it is a zero divisor and hence $E F=0$. If $E F=0$, then, since $\|E\|=\|F\|=1, E$ and $F$ are non-zero zero divisors, which contradicts the hypothesis.

Definition. We say that multiplication is bounded below on a subset $\mathcal{S}$ of a Banach algebra if there is an $\varepsilon>0$ such that $\|A B\| \geq \varepsilon\|A\|\|B\|$ for all $A$ and $B$ in $\mathcal{S}$.

Corollary 2. Let $\mathcal{S}$ be a semigroup of linear transformations on a finite-dimensional space, on which multiplication is bounded below. If there exists a $k$ such that

$$
\begin{aligned}
& r(A B) \leq k r(A) r(B) \quad \text { for all } A \text { and } B \text { in } \mathcal{S} \text {, then } \\
& r(A B) \geq \frac{1}{k} r(A) r(B) \quad \text { for all } A \text { and } B \text { in } \mathcal{S} .
\end{aligned}
$$

Proof. Let $\mathcal{T}$ be the closure of

$$
\{t S: t \geq 0, S \in \mathcal{S}\}
$$

Then the inequality of the hypothesis extends to the semigroup $\mathcal{T}$ so the corollary will follow from Theorem 2 if we show that $\mathcal{T}$ has no non-zero zero divisors. Note that

$$
\|A B\| \geq \varepsilon\|A\|\|B\|
$$


for all $A$ and $B$ in $\mathcal{S}$ implies the same for all $A$ and $B$ in $\mathcal{T}$, so $\mathcal{T}$ does not have non-zero zero divisors.

Corollary 3. If multiplication is bounded below on a semigroup $\mathcal{S}$ of linear transformations on a finite-dimensional space, and if spectral radius is submultiplicative on $\mathcal{S}$, then spectral radius is multiplicative on $\mathcal{S}$.

Proof. $\frac{1}{1}=1$.

There is a result analogous to Theorem 2 for the reverse inequality.

Theorem 3. Let $\mathcal{S}$ be a semigroup of linear transformations on a finite-dimensional space which is closed under multiplication by positive real numbers and is also closed in the topological sense. If $\mathcal{S}$ has no non-zero zero divisors and there is an $\varepsilon>0$ such that $r(A B) \geq \operatorname{\varepsilon r}(A) r(B)$ for all $A$ and $B$ in $\mathcal{S}$, then $r(A B) \leq \frac{1}{\varepsilon} r(A) r(B)$ for all $A$ and $B$ in $\mathcal{S}$.

Proof. It suffices to show that there is some $k$ such that $r(A B) \leq k r(A) r(B)$ for all $A$ and $B$ (by Theorem 11). If not, then for every $k$ there are $A_{k}$ and $B_{k}$ in $\mathcal{S}$ satisfying

$$
r\left(A_{k} B_{k}\right)>k r\left(A_{k}\right) r\left(B_{k}\right)
$$

with $r\left(A_{k}\right)$ and $r\left(B_{k}\right) \neq 0$. For each $k$, let $C_{k}=\frac{A_{k}}{r\left(A_{k}\right)}$ and $D_{k}=\frac{B_{k}}{r\left(B_{k}\right)}$.

If $\left\{C_{k}\right\}$ was not bounded in norm, $\left\{\frac{C_{k}}{\left\|C_{k}\right\|}\right\}$ would have a subsequence which converged to a linear transformation of norm 1 and spectral radius 0 , contradicting the lack of zero divisors in $\mathcal{S}$. Thus $\left\{C_{k}\right\}$ and $\left\{D_{k}\right\}$ are bounded sequences, and so therefore is $\left\{C_{k} D_{k}\right\}$. But $r\left(C_{k} D_{k}\right)>k$ for each $k$, which is a contradiction.

Theorem 3 has corollaries similar to those of Theorem 2.

\section{Semigroups of COMPaCt operators}

The results of section 3 do not appear to extend to semigroups of compact operators. However, a theorem of [1] can be extended to give a result in the case of irreducible semigroups (i.e., semigroups with no non-trivial invariant (closed) subspaces).

Theorem 4. If $\mathcal{S}$ is an irreducible semigroup of compact operators on Hilbert space and if there exists $k>0$ such that $r(A B) \leq k r(A) r(B)$ for all $A$ and $B$ in $\mathcal{S}$, then $r(A B) \geq \frac{1}{k} r(A) r(B)$ for all $A$ and $B$ in $\mathcal{S}$.

Proof. The proof is a very small modification of the proof of the case $k=1$ given in Theorem 2.1 of [1]. As in [1, we assume that $\mathcal{S}$ is closed under multiplication by positive numbers and is also closed in the topological sense. Also as in [1, we first consider the case where $A^{2}=A$ and $B^{2}=B$ and $r(A)=r(B)=1$. Then

$$
r(A B)=r\left(A^{2} B^{2}\right)=r(B A A B) \leq k(r(A B))^{2} .
$$

So, since $r(A B) \neq 0$ (as in [1]), it follows that

$$
\frac{1}{k} \leq r(A B)
$$

For the general case where $r(A)=r(B)=1$, choose, as in [1], increasing sequences of positive integers and scalars $a$ and $b$ of modulus 1 such that $\left\{a A^{n_{j}}\right\} \rightarrow P$ and $\left\{b B^{n_{j}}\right\} \rightarrow Q$ with $P$ and $Q$ non-zero idempotents. 
Following [1] but inserting " $k$ " gives

$$
\begin{aligned}
r\left(A^{n_{j}} B^{n_{j}}\right) & =r\left(A B B^{n_{j}-1} A^{n_{j}-1}\right) \\
\leq & k r(A B) r\left(B^{n_{j}-1} A^{n_{j}-1}\right) \\
\leq & k^{2} r(A B) r\left(B^{n_{j}-1}\right) r\left(A^{n_{j}-1}\right) \\
& =k^{2} r(A B) .
\end{aligned}
$$

Taking limits yields

$$
r(P Q) \leq k^{2} r(A B) .
$$

Since $\frac{1}{k} \leq r(P Q)$ by the first part of this proof, it follows that

$$
\frac{1}{k^{3}} \leq r(A B) \text {. }
$$

This holds for all $A$ and $B$ in $\mathcal{S}$ of spectral radius 1 . Thus

$$
\frac{1}{k^{3}} r(A) r(B) \leq r(A B) \leq k r(A) r(B)
$$

for all $A$ and $B$ in $\mathcal{S}$. Theorem 1 then gives $\frac{1}{k} r(A) r(B) \leq r(A B)$.

\section{An example}

Let $\mathcal{S}$ be the semigroup consisting of all positive integral multiples of

$$
A=\left(\begin{array}{ll}
2 & 0 \\
1 & 0
\end{array}\right), \quad B=\left(\begin{array}{ll}
0 & 2 \\
0 & 1
\end{array}\right), \quad C=\left(\begin{array}{ll}
1 & 0 \\
2 & 0
\end{array}\right), \quad D=\left(\begin{array}{ll}
0 & 1 \\
0 & 2
\end{array}\right) .
$$

Direct multiplication shows that $\mathcal{S}$ is a semigroup, and the inequalities

$$
\frac{1}{4} r(E) r(F) \leq r(E F) \leq 4 r(E) r(F)
$$

hold and are sharp for $\mathcal{S}$.

\section{REFERENCES}

1. W. Longstaff and H. Radjavi, On permutability and submultiplicativity of spectral radius, Can. J. Math. 47 (1995), 1007-1022. MR 97d:47005

Department of Mathematics, University of Ljubljana, 1000 Ljubljana, Slovenia

E-mail address: matjaz.omladic@un-lj.si

Department of Mathematics, Dalhousie University, Halifax, Nova Scotia, Canada B3H $4 \mathrm{H} 8$

E-mail address: radjavi@mscs.dal.ca

Department of Mathematics, University of Toronto, Toronto, Ontario, Canada M5S $3 \mathrm{G} 3$

E-mail address: rosent@math.toronto.edu

Department of Mathematics, University of Victoria, Victoria, British Columbia, CANAdA V8W 3P4

E-mail address: asourour@alpha.uvic.ca 